\title{
OPTICS DIAGNOSTICS AND TUNING FOR LOW EMITTANCE BEAM IN KEK-ATF DAMPING RING
}

\author{
H.Hayano, S.Kamada, K.Kubo, T.Naito, K.Oide, N.Terunuma, N.Toge, J.Urakawa, KEK; \\ S.Kashiwagi, Graduate U. for Advanced Studies; T.Okugi, Tokyo Metropolitan U.; M.Takano, Toho \\ U.; K.Bane, T.Kotseroglou, M.Minty, M.Ross, J.Turner, M Woodley and F.Zimmermann, SLAC
}

\section{Abstract}

The target of KEK-ATF is producing electron beam with vertical emittance of $0.01 \mathrm{~nm}-\mathrm{rad}\left(1 \times 10^{-11} \mathrm{~m}-\mathrm{rad}\right)$. Corrections of the dispersions are essential to achieve the low emittance. Because the actual optics of the beam line should be known for these corrections, beam based optics diagnostics has been performed. Simulations showed the validity and the accuracy of the method. Effects of skew quadrupoles have also been studied.

\section{OPTICS DIAGNOSTICS}

In the early stage of the operation of the ATF Damping Ring, there were big discrepancies between observations of beam behavior and calculations based on our optics model. In order to make the model more accurate, optics diagnostics was performed. The method were reported with results of its test before[1].

Errors of quadrupole strengths of the quadrupole magnets and the combined bending magnets have been estimated using beams, by steering beam and measuring the orbit in down stream. There are about 100 steering magnets and 100 BPMs in the ring and the measured response coefficients $\left(\mathrm{R}_{12}\right.$ and $\left.\mathrm{R}_{34}\right)$ at the BPMs from the steering magnets are fit by errors of strength of quads, BPMs and steerings. Magnets are divided into 10 types and we assumed that each type of the magnets has a common error ratio because they have the same design. (See Table 1.) In order to correct the original model, which is based on magnetic field measurements, 'fudge factor' has been introduced for each type as relative error of the quadrupole field strength.

The diagnostics have been performed several times, especially when the optics setting was changed or beam position monitor (BPM) system was modified.

Results from the most recent measurement are shown in Fig. 1 and Table 1 which was taken in November 1998 after improvement of the BPM electronics [3]. To avoid nonlinear effects, sextupole magnets were turned off. Because a part of beam was lost far downstream for some settings, BPMs only in $30 \mathrm{~m}$ downstream changed steering magnets were used for the analysis. 10 BPMs in the wiggler sections, which had big non-linear response were not used. Fig. 1(a) shows response coefficients from the steering magnets to the BPMs calculated using the original model without 'fudge' factors vs. measured coefficients. Fig. 1(b) shows the model with new 'fudge' factors vs. measured coefficients. The new fudge factors are listed in Table 1 . The new model has different strength of quadrupole fields from the original model up to a few percent.
Table 1: Magnet types and fudge factors

\begin{tabular}{|c|c|c|c|}
\hline Type & $\begin{array}{r}\text { Number } \\
\text { of Magnets }\end{array}$ & $\begin{array}{l}\text { Fudge } \\
\text { factors }\end{array}$ & \\
\hline BH1R-N & 6 & $-2.07 E-3$ & \multirow{3}{*}{$\begin{array}{l}\text { Combined } \\
\text { bend* }\end{array}$} \\
\hline BH1R-Ma & 16 & $2.87 \mathrm{E}-2$ & \\
\hline BH1R-Mb & 14 & $1.58 \mathrm{E}-2$ & \\
\hline $\begin{array}{l}\text { QF2R } \\
\text { (thick) }\end{array}$ & 26 & $1.11 \mathrm{E}-3$ & $\begin{array}{c}\text { Main Quad } \\
\text { in Arc Section }\end{array}$ \\
\hline H2 (thin) & 8 & $2.20 \mathrm{E}-2$ & \multirow{2}{*}{$\begin{array}{l}\text { Matching Quad } \\
\text { in Arc Section }\end{array}$} \\
\hline H3 (thick) & 8 & $-8.94 \mathrm{E}-3$ & \\
\hline H4 (thick) & 16 & $-1.04 \mathrm{E}-2$ & \multirow{4}{*}{$\begin{array}{c}\text { Matching Quad } \\
\text { in Straight } \\
\text { Section }\end{array}$} \\
\hline $\mathrm{T} 1$ (thin) & 4 & $-1.95 \mathrm{E}-2$ & \\
\hline $\begin{array}{l}\mathrm{T} 42 \mathrm{~L} \\
\text { (thick) }\end{array}$ & 4 & $5.47 \mathrm{E}-3$ & \\
\hline T42S(thin) & 6 & $1.01 \mathrm{E}-2$ & \\
\hline
\end{tabular}

*BH1R-N, -Ma, -Mb have the same spec. but made by different companies or from different lots.
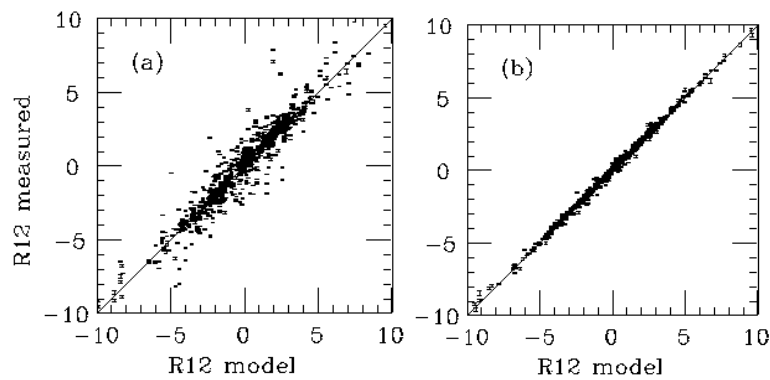

Fig. 1: Response coefficients from the steering magnets to the BPMs. (a):The original model vs. measurement and (b) corrected model vs. measurement. Both horizontal and vertical coefficients are plotted.

After the diagnostics, model calculations for orbit corrections and dispersion corrections have become useful in our beam tuning.

Simulations were done to estimate the accuracy of the method. The measurement of orbit change at BPMs changing steerings was simulated using the computer code SAD [2]. In the simulation, errors were set as in Table 2.

Table 2: Random errors in optics diagnostics simulation.

\begin{tabular}{|c|c|}
\hline BPM resolution (pulse to pulse) & gaussian r.m.s. $10 \mu \mathrm{m}$ \\
\hline BPM position sensitivity factor & gaussian r.m.s 10\% \\
\hline Steering's kick angle / current & gaussian r.m.s 10\% \\
\hline Quadrupole field(thick magnets) & Uniform $+-1 \%$ \\
\hline Quadrupole field(thin magnets) & Uniform $+-2 \%$ \\
\hline
\end{tabular}



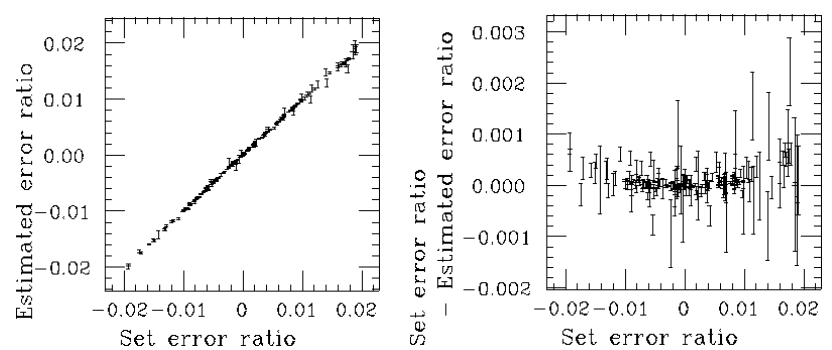

Fig 2 : Set error ratio vs. (left) estimated error ratio of the strength of quadrupole field and (right) difference between the estimated error ratio and the set error ratio. Results of all types from 22 different random seeds are plotted. The error bars are the estimated resolution.

The errors of position sensitivity factors and the kick angles seem over estimated but further simulation studies show that the fitting results of quadrupole field errors are not sensitive to these errors. Lengths of the quadrupole magnets are $18 \mathrm{~cm}$ (thick) or $6 \mathrm{~cm}$ (thin). We assumed the thick magnets have less error because their effective lengths are expected to be better known. The combined bending magnets were treated as thick magnets and BH1R-Ma and $\mathrm{BH} 1 \mathrm{R}-\mathrm{Mb}$ are regarded as the same type in this simulation.

BPM data were simulated from calculated beam positions considering the BPM resolution. 5 different currents, I=-1, $-0.5,0,0.5,1 \mathrm{~A}$, were set for every steering magnets where 1 A corresponds to the kick angle of about $0.3 \mathrm{mrad}$. Optics was set as a design which is the base of the present operation optics.

The errors of quadrupole fields, the sensitivity factors of the BPM and the current-to- angle factors of the steering magnets were estimated using the same analysis program for the real data. Simulations were done with 22 random seeds.

The left of Fig. 2 shows set error ratio vs. estimated error ratio of the quadrupole field strengths of all types. Results from all 22 random seeds are plotted together. The right shows the difference of estimated error ratio and the set error ratio. The error bars are the estimated resolution propagated from the resolution of BPMs, 10 micron. Most of the estimations are consistent with the set values within the resolutions indicating the validity of this analysis. Fig. 3 shows the resolution for each type of magnets averaged over the 22 random seeds.

The result shows that the common error for the same type of magnets is expected to be estimated in good resolution.

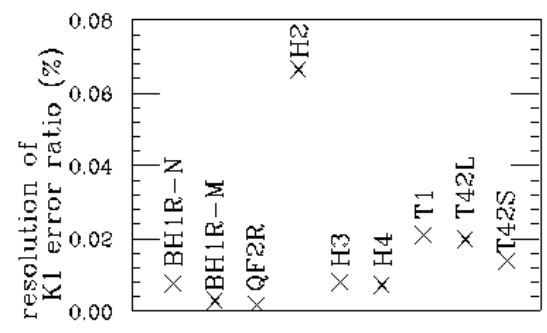

Fig. 3 : Estimated resolution of strength ratio for each type of magnets $(\%)$ averaged over the 22 random seeds.

\section{TUNING FOR LOW EMITTANCE}

\subsection{Dispersion Correction}

It is essential to make the vertical dispersion small in the arc sections for producing low vertical emittance beams. Relation between the dispersion and emittance was simulated using SAD [2]. In the simulation, all magnets were misaligned randomly with r.m.s. 20 micron. The vertical dispersions at the BPMs were tried to be zero using the steering magnets. Simulation was done for 2000 different random seeds. Fig. 4 shows square of the vertical dispersion averaged over the BPMs in the arc section vs. the vertical emittance calculated by SAD. Other effects such as the intrabeam scattering were not considered. One point corresponds to one random misalignment. The emittance has linear dependence on the square of the vertical dispersion at BPMs as

$$
\varepsilon_{y}(\mathrm{~nm}-\mathrm{rad}) \approx 0.0006\left\langle\eta_{y, B P M}^{2}\left(\mathrm{~mm}^{2}\right)\right\rangle_{\text {arc }}
$$

where \langle\rangle$_{\text {arc }}$ means the average in the arc sections.

Though we assumed the random misalignment of 20 micron, results of further simulations with other realistic misalignment assumptions give almost the same dependence as the equation (1).

The result shows that the vertical dispersion at BPMs should be about $4 \mathrm{~mm}$ eto achieve our goal, $0.01 \mathrm{~nm}$-rad.

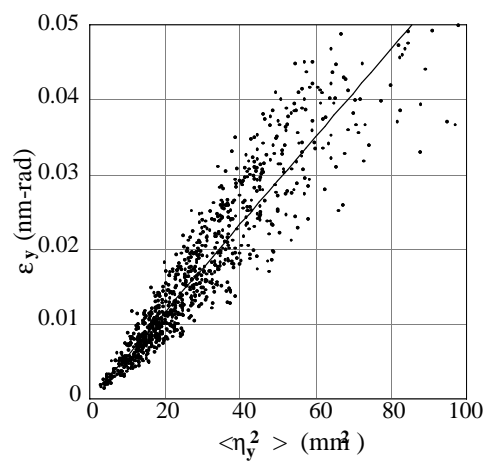

Fig. 4 : Square of the vertical dispersion averaged over BPMs in the arc section vs. the vertical emittance from simulation.

Dispersion function at BPMs in the ring are measured as the orbit difference for different RF frequencies.

A dispersion correction routine has been developed. Set of vertical steerings is calculated to reproduce the measured dispersion in the perfect model and opposite of the steerings are set. An typical example is shown in Fig. 5, the vertical dispersion before and after the correction. In this example, r.m.s. of the vertical dispersion in the arc sections are 13 $\mathrm{mm}$ before the correction and $4.3 \mathrm{~mm}$ after the correction, from the equation (1), corresponding to the vertical emittances of 0.10 and $0.011 \mathrm{~nm}$-rad. On the other hand, apparent emittances, roughly monitored using SR- 
interferometer [4], were about 0.08 and $0.05 \mathrm{~nm}$-rad before and after the correction, respectively. The reason of the big difference in the case of after the correction has not been known yet. Both the emittance measurement and the dispersion measurement should be studied more.

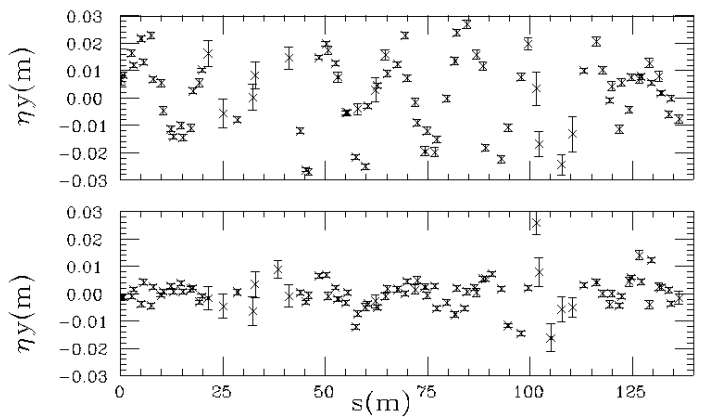

Fig. 5 : Measured vertical dispersion before (top) and after the correction (bottom).

\subsection{Local Orbit Bump}

Vertical orbit bumps have been applied in the ring to reduce the vertical emittance in try-and-error monitoring the vertical beam size using the SR-interferometer [4]. Because the dispersion correction program assumes no strength errors and no misalignment of magnets, the calculation can not be perfect and this empirical method is sometimes effective.

\subsection{Effect of Skew Quadrupole Fields}

Trim coils of 4 sextupole magnets are connected to produce skew quadrupole fields though there are not skew quadrupole magnets. The maximum strength of the field $\left(\mathrm{SK}_{1}\right)$ is estimated to be about $0.033 \mathrm{~m}^{-1}$ for each magnet. We have tried to see the effects of the skews to the vertical emittance but no clear effect has been observed so far.
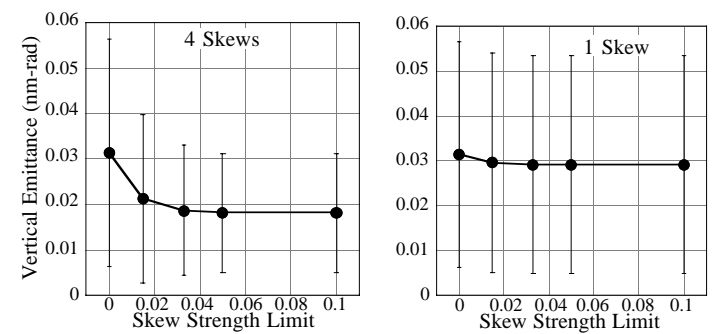

Fig 6 : Average of emittance vs. the limit of the strength. From simulation with 100 random seeds, the error bars represent r.m.s. fluctuation.

Fig. 6 shows result of simulation of effects of the skews. All magnets had random alignment errors of r.m.s. 30 micron and rotation error r.m.s. $0.5 \mathrm{mrad}$. Orbit and dispersion corrections were applied and strength of skew fields were searched for the minimum vertical emittance assuming the emittance was precisely monitored. The figure shows the average and the r.m.s. fluctuation of 100 random seeds vs. the limit of the strength of the skews. The left figure shows the case using all 4 skews and the right shows the case using only 1 skew. The results show that the present limit, $0.033 \mathrm{~m}^{-1}$, is large enough, the effect of 1 skew will not be seen but proper set of 4 skews can reduce the emittance. As shown in Fig. 7, the emittance can be reduced more using more number of skews. The trim coils of the all sextupole magnets will be connected for skew quadrupole fields and the effects will be tested soon. However to find a good setting of the skews is not straight forward and we need good emittance monitors in order to try many settings.

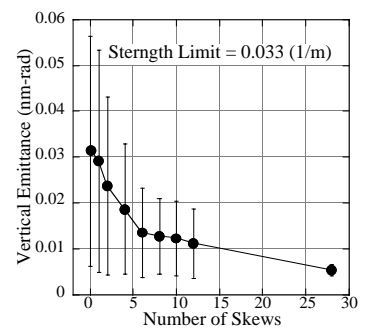

Fig. 7: Emittance vs. number of skews. From simulation with 100 random seeds, the error bars represent r.m.s. fluctuation.

\section{SUMMARY}

First order optics diagnostics was performed and the model calculations have been improved. The validity of the diagnostics method was demonstrated and the accuracy was estimated by simulations. Methods for tuning for the low vertical emittance, dispersion correction, local bumps and skew quadrupole fields, were discussed. The dispersion correction worked well and the apparent vertical dispersion seemed to be close to the goal for the vertical emittance, $0.01 \mathrm{~nm}-\mathrm{rad}$. But the apparent vertical emittance was still large $[5,6]$ and more study of both the dispersion and the emittance measurement will be necessary. Effects of skew quadrupole fields have not been observed yet. The simulation showed that the effects will not be seen easily but it is possible to use the set of skews for the low emittance tuning with good emittance monitors.

\section{ACKNOWLEDGMENT}

The authors would like to thank professors Sugawara, Kihara and Takata for continuous support and encouragement.

\section{REFERENCES}

[1] K. Kubo et. al., "Test of Optics Diagnostics in ATF”, PAC'97, Vancouver, May 1997.

[2] http://www-acc-theory.kek.jp/SAD/sad.html

[3] M. Takano et. al., "BPM electronics upgrade for ATF DR", EPAC98, Stockholm, June, 1998.

[4] T. Mitsuhashi et. al., "Beam size measurement at ATF DR by the use of SR interferometer", EPAC98.

[5] T.Naito et. al., "Emittance Measurement at ATF Damping Ring", this conference.

[6] T.Okugi et. al., "Vertical Emittance in the KEK Accelerator Test Facility", this conference. 